\title{
CAPITOLO 2
}

\section{ArcheOS e-learning project}

\author{
Alessandro Bezzi*, Luca Bezzi ${ }^{*}$, Rupert Gietl', \\ Wilfrid Allinger-Csollich ${ }^{\dagger}$, Sandra Heinsch ${ }^{\dagger}$, \\ Walter Kuntner ${ }^{\dagger}$
}

Sommario. Con il seguente contributo si intende presentare un progetto sperimentale che nasce dalla collaborazione dell'Università di Innsbruck (Dipartimento di Orientalistica e Storia Antica) e la società Arc-Team snc. Tale progetto prevede la creazione di una serie di tutorial riguardanti l'utilizzo di software libero in archeologia. Ogni tutorial si basa sull'esperienza concreta dello scavo scuola di Aramus (Armenia).

ABSTRACT. In this paper we would like to present an experimental project born from the collaboration of the University of Innsbruck (Department of Near East and Ancient History) and Arc-Team. Its main purpose is to create some tutorilals about the use of Free Software in archaeology. Each tutorial is based on the real experience of the "Aramus Excavations and Field School".

\section{Lo scavo di Aramus}

La "Aramus Excavations and FieldSchool" è un progetto di scavo condotto dall'Università di Innsbruck ${ }^{1}$ in collaborazione con l'Università di Yerevan ${ }^{2}$ e l'Accademia Nazionale di Scienza della Repubblica d'Armenia ${ }^{3}$. Il sito, scelto in seguito ad una campagna di survey condotta nel corso del 2003, si trova su di un'altura naturalmente protetta che emerge sull'altopiano del Kotayk 
(Armenia), a circa $15 \mathrm{Km}$ dalla capitale Yerevan, ad un'altitudine di $1450 \mathrm{~m}$ sul livello del mare.

Le prime indagini furono condotte dal Lalayan e dal Bajburtyan alla fine degli anni 1920 in un contesto di ricognizioni archeologiche generali svolte per la catalogazione dei siti preistorici della regione 4 . I primi sondaggi furono effettuati invece alla fine degli anni 60 e inizio 70 dalla Khanzadyan con l'intento di completare il quadro storico-culturale della Prima Età del Ferro locale (1000-800 a. C.) che stava emergendo negli scavi sistematici effettuati sul sito di Elar ${ }^{5}$, situato a soli $3 \mathrm{Km}$ a occidente di Aramus, nelle fortezze e necropoli nella zone costiera settentrionale del lago di Sevan, e infine a Metsamor e a Artik situate rispettivamente nella piana dell'Ararat e sull'altopiano di Shirak ${ }^{6}$.

Gli scavi condotti nel 1988 dall'Avetysian nella parte centrale della fortezza, dove fu scoperta una porta monumentale rafforzata da due torri che dava accesso ad una serie di stanze allineate lungo i muri di fortificazione, portarono ad uno spostamento della data di fondazione. Insieme a cocci di ceramica d'impasto nero con decorazioni di lisciatura geometrica sulla superficie, tradizionalmente attribuite alla Prima Età del Ferro, furono trovati nei livelli di fondazione un gran numero di frammenti d'impasto rosso levigato di tipico stampo Urarteo ${ }^{7}$. La fortezza di Aramus deve quindi essere considerata una fondazione urartea della Seconda Età del Ferro che ben si pone nel contesto di espansione militare urartea volta alla conquista delle regioni a ridosso del lago di Sevan voluta da Argisti I (785 - 763 a. C.) e da Rusa I (735 - 714 a. C. $)^{8}$.

Gli scavi armeno-austriaci iniziati nel 2004 nella parte orientale della fortezza rivelarono ben presto che l'occupazione delle strutture fortificate continuò ben oltre la caduta dell'impero Urarteo, avvenuta intorno alla metà del VII secolo a.C $\mathrm{C}^{9}$. Le ultime tracce di frequentazione risalgono infatti al IV secolo a. C. e mostrano una continuità della cultura materiale nettamente collegata alla tradizione del Primo Ferro che si esprime parallelamente a forme di vasellame ed impasti che possono essere definiti achemenidi. Gli scavi, che da allora si sono estesi su tutta l'area dell'abitato, confermarono che la fortezza di Aramus non subì né distruzioni violente né fasi di abbandono in seguito alla caduta dell'impero Urarteo, bensì un rafforzamento durante il periodo achemenide ed un mantenimento della sua massima estensione per tutto il VI e V secolo a. C., dopodiché iniziò un graduale ridimensionamento dell'abitato sulle parti fortificate poste a ridosso della cresta della collina ${ }^{10}$.

Dal punto di vista archeologico il progetto intende fare luce, a livello regionale, sulla crescita e il declino del regno di Urartu, in relazione alle strutture sociali e politiche che lo precedettero e che lo seguirono, e sulla qualità intrinseca al fenomeno Urartu inteso come prima manifestazione politica di un'unità materiale nella storia della regione transcaucasica. I risultati ottenuti sottolineano l'importanza di spostare l'attenzione delle indagini archeologiche 
sulle culture autoctone nel Caucaso Minore per poter meglio concettualizzare lo sviluppo delle caratteristiche della cultura materiale del Ferro e la loro interazione con le strutture politiche di Urartu e degli Achemenidi.

Un secondo aspetto del progetto "Aramus Excavations and FieldSchool" è la sua configurazione come scavo scuola universitario che, tramite la didattica perseguita durante le missioni annuali, mira alla formazione di archeologi in un contesto complessivamente definibile "Free/Libre". Il corso universitario integra attivamente le conoscenze che lo studente acquisisce sul campo, accompagnandolo attraverso le singole fasi di ricerca ed interpretazione del dato archeologico. La FieldSchool è dunque un'esperienza concreta di scavo stratigrafico e di documentazione informatizzata delle evidenze antropiche emerse. Nel 2006 questo secondo aspetto è stato curato dalla società Arc-Team che si è avvalsa esclusivamente di software libero $\left(\mathrm{ArcheOS}^{11}\right)$, situazione che ha permesso di porre le basi del progetto presentato in questo articolo.

\section{Aspettative}

L'obiettivo prefissato per quanto riguarda lo sviluppo della componente didattica della FieldSchool prevede la realizzazione del progetto DADP (Digital Archaeological Documentation Project ${ }^{12}$ ), una serie di tutorial riguardanti l'utilizzo di software libero in campo archeologico. L'idea di fondo, nonché la principale aspettativa, vorrebbe il commutarsi di questo insieme iniziale di lezioni su singoli argomenti (sono previsti testi, video-tutorial e video interattivi) in uno strumento didattico libero e aperto, aggiornabile ed integrabile liberamente (sistema wiki). Una volta terminato il livello didattico "base" (sono previsti esercizi organizzati su tre livelli di apprendimento: base, intermediate, advanced), tale strumento dovrebbe entrare a pieno titolo nell'insegnamento del corso di studio in Storia del Vicino Oriente Antico presso l'Università di Innsbruck.

Ogni singolo documento ed ogni dato relativo agli esercizi sarà rilasciato attraverso una licenza libera che preveda la possibilità di integrare e aggiornare il tutto con nuovi contributi di altri autori, sperando nella partecipazione di studenti e colleghi interessati. Il nostro più grande auspicio è che questo esperimento fornisca uno stimolo, all'interno del mondo scientifico, per spingere verso una sempre maggiore condivisione di dati, idee e strumenti.

\section{Un'esperienza di condivisione}

Abbiamo pensato di presentare in questa sede la nostra esperienza in quanto si tratta essenzialmente di un'esperienza di condivisione, argomento centrale quando si parla di software libero. Ci siamo infatti trovati più volte ad analizzare questa tematica sotto almeno tre aspetti: 
(1) la condivisione degli strumenti (nel nostro caso il software libero e nello specifico ArcheOS);

(2) la condivisione della conoscenza (alla quale cerchiamo di arrivare per mezzo del progetto DADP);

(3) la condivisione dei dati (problema generalmente molto sentito in archeologia).

Nei prossimi paragrafi vorremmo descrivere brevemente la situazione attuale per quanto riguarda questi tre livelli di condivisione.

\subsection{La situazione attuale nella condivisione degli strumenti}

Nel prendere in esame la problematica della condivisione degli strumenti (i software), non abbiamo avuto particolari difficoltà, in quanto si tratta di un argomento che Arc-Team ha affrontato già da qualche tempo all'interno del progetto OpArc ${ }^{13}$. Uno degli obbiettivi raggiunti da tale progetto è la creazione di ArcheOS (un sistema operativo basato su GNU/Linux e corredato da software libero selezionato espressamente per la ricerca archeologica). Partendo da queste premesse non è stato difficile "liberarsi" dal software chiuso, vista anche la grande quantità e l'ampia scelta presente nel mondo FS/OS.

ArcheOS è infatti il sistema operativo utilizzato nella campagna di scavo di Aramus 2006. Durante quella spedizione la nostra scelta a favore del software libero è stata premiata sia durante la spedizione grazie ad una maggiore stabilità rispetto ai programmi utilizzati precedentemente (i computer con il sistema operativo cui si era fatto ricorso nella campagna del 2005 era inutilizzabile l'anno seguente a causa dell'enorme numero di virus, spyware e malware in generale), sia nella fase successiva di post-scavo grazie agli ottimi risultati raggiunti (a livello informatico).

In definitiva la situazione attuale riguardo la condivisione degli strumenti è molto incoraggiante proprio perché si dispone di un'ampia scelta di programmi liberi protetti da solide licenze (ad esempio la GPL). L'unico inconveniente, sebbene di anno in anno si stia notevolmente ridimensionando, si riferisce allo stadio di sviluppo ancora "embrionale" di alcuni programmi pertinenti ai campi più settoriali della ricerca.

\subsection{La situazione attuale nella condivisione della conoscenza}

Se si analizza lo status quo relativo alla condivisione della conoscenza (che è l'argomento centrale del nostro intervento), il panorama cambia leggermente. Infatti, se è vero che spesso disponiamo di un'abbondante documentazione riguardante i FS/OS (e non di rado rilasciata essa stessa con licenza libera FDL), 
è altrettanto vero che manca una documentazione che prenda in esame l'applicazione di questi software al campo archeologico (e questa è proprio la lacuna che speriamo di colmare con il progetto DADP). A nostro parere la mancanza di testi specifici è l'ostacolo maggiore per l'adozione del software libero in archeologia e pensiamo (o meglio speriamo) che risolvendo questo problema si possa dare una spinta determinante alla loro diffusione.

\subsection{La situazione attuale nella condivisione dei dati}

Infine la situazione attuale riguardo la condivisione di dati sembra essere la nota più desolante per vari motivi. In primo luogo esistono pochi esempi di effettiva condivisione di dati archeologici e spesso riguardano paesi esteri. In secondo luogo ogni paese fa riferimento a legislazioni differenti in materia di beni culturali.

Il problema della condivisione dei dati appare quindi il più complesso. Un possibile spunto per un'ipotetica soluzione di una situazione tanto complicata potrebbe essere la creazione di una licenza specifica per i beni culturali e archeologici. Una licenza del genere dovrebbe essere redatta sulla traccia delle altre licenze libere (GPL, FDL, CC, ecc...), ma probabilmente dovrebbe avere delle peculiarità tutte sue, soprattutto per garantire a chiunque l'accessibilità ai dati archeologici troppo spesso soggetti all'arbitrarietà di chi ne è in possesso ${ }^{14}$.

\section{Le problematiche affrontate}

Per entrare più nello specifico del progetto qui presentato, descriveremo ora i principali problemi cui si è andati incontro e le soluzioni che si sono via via adottate.

\subsection{Problemi relativi alla condivisione degli strumenti}

Abbiamo già accennato che per la realizzazione del progetto DADP ci siamo basati sulla distribuzione ArcheOS, principalmente per poter garantire agli studenti-utenti di lavorare su di un sistema operativo sviluppato appositamente per il mondo dell'archeologia. Abbiamo inoltre visto che uno dei pochi problemi riguardanti la migrazione ai FS/OS è il ritardo che si registra in determinati ambiti (generalmente settoriali) rispetto ad alcune applicazioni proprietarie. Nella nostra esperienza abbiamo cercato di oltrepassare questo ostacolo utilizzando una combinazione di diversi programmi per ottenere lo stesso risultato che, a livello di software proprietario, si ottiene con un'applicazione stand-alone. I risultati ottenuti sono stati superiori alle aspettative. 
A titolo di esempio riportiamo il caso relativo alla creazione di fotomosaici georeferenziati. Nel mondo del software chiuso, o dello shareware, esistono come dicevamo applicazioni stand-alone (Monobild@ , RDF@, ecc...) per ottenere il raddrizzamento e la georeferenziazione delle immagini. Lo stesso procedimento si potrebbe ottenere utilizzando esclusivamente GRASS, ma la rigidità del programma, dovuta ad esigenze di precisione, richiede un numero troppo elevato di marche (nove) per le tempistiche di un cantiere archeologico (dove i tempi di raccolta dati sono generalmente ristretti). Di conseguenza, per la campagna di Aramus 2006 è stato messo a punto e testato da Arc-Team, un sistema che combina GRASS, e-foto e GIMP. Senza entrare nei particolari (eccessivamente lunghi da descrivere ${ }^{15}$ ), basti dire che sebbene il procedimento si discosti dalle procedure standard, i risultati ottenuti hanno superato in qualità i prodotti commerciali grazie all'indiscusso vantaggio di equalizzazione della luce tra le diverse foto.

\subsection{I problemi relativi alla condivisione della conoscenza}

Per quanto riguarda i tutorial, e quindi la condivisione della conoscenza, il progetto DADP prevede la creazione di una serie di esempi di applicazione pratica di FS/OS in archeologia. La difficoltà iniziale relativa alla scelta del formato e del mezzo attraverso cui divulgare i tutorial si è positivamente risolta, dopo una serie di prove (fallimentari) con vari formati di testo, con l'adozione di un sistema wiki. Si tratta di un sistema testato e sicuro (si pensi a Wikipedia), che permette la condivisione non solo di testi, ma soprattutto di immagini, file e filmati ${ }^{16}$. Inoltre è semplice ed intuitivo, oltre a presentare l'indiscusso vantaggio di poter essere aggiornato e corretto in tempo reale (un esempio è il tutorial riguardante la creazione di fotomosaici georeferenziati, che è stato più volte migliorato anche in corso di scrittura). Tutti questi vantaggi non sono riscontrabili in un semplice testo scritto, sia esso in formato digitale o su carta stampata. Inoltre un sistema wiki, attraverso la rete, può raggiungere un maggior numero di utenti-autori rispetto a qualsiasi pubblicazione cartacea. La forza principale di un sistema wiki è infatti la comunità che vi sta alla base.

\subsection{I problemi relativi alla condivisione dei dati}

In ultimo affrontiamo il problema, tuttora irrisolto, della condivisione dei dati. Si tratta di un passo obbligato, in quanto nell'ottica dei tutorial, gli esercizi proposti devono essere replicati dall'utente del DADP. Grazie alla disponibilità dell'Università di Innsbruck è stato possibile selezionare, utilizzare e quindi condividere i dati della campagna di Aramus. Bisogna però fare una precisazione: in questo caso la nostra esperienza non può essere considerata come un esempio di condivisione di dati nel panorama italiano, in quanto si riferisce alla realtà universitaria austriaca. Il problema principale per quanto riguarda 
la condivisione di dati archeologici rimane (a nostro avviso) quello della mancanza di una licenza specifica, che garantisca il libero accesso ai dati anche nel caso si tratti di materiale non ancora pubblicato (si potrebbe pensare a particolari restrizioni per questo genere di situazioni). A tale riguardo speriamo che si possa affrontare questo argomento all'interno dei prossimi incontri del workshop su "Open Source, Free Software e Open Format nei processi di ricerca archeologica", magari in un'ottica internazionale.

Un'altra problematica inerente la condivisione dei dati, riguarda la tipologia dei dati da condividere. In sostanza bisognerebbe considerare se nell'ambito di un progetto archeologico di scavo (ma non solo) sia il caso di condividere anche i dati grezzi, oppure solamente quelli elaborati. A livello teorico una buona documentazione per essere divulgata dovrebbe rappresentare una semplificazione della realtà analizzata, dovrebbero cioè essere filtrate tutte quelle informazioni (raccolte in via precauzionale, ma rivelatesi non necessarie) che creano un elemento di disturbo alla lettura della situazione registrata. Sotto questo aspetto sarebbe quindi sufficiente condividere solo i dati elaborati, essendo elevato il rischio che i dati grezzi creino confusione. Una scelta del genere precluderebbe però a chiunque la possibilità di controllo sulle conclusioni raggiunte, impedendo quindi che vengano formulate ipotesi alternative. I dati elaborati potrebbero cioè fornire una visione non oggettiva (ma già interpretata) della realtà indagata.

A tale proposito il parere di chi scrive è che sia consigliabile comunque condividere anche i dati grezzi, non solo per favorire un eventuale dibattito scientifico, ma anche per fornire la possibilità a chiunque sia interessato di ripercorrere le tappe e i processi mentali che hanno portato alle conclusioni presentate, evitando così di richiedere una sorta di "atto di fede" riguardo al proprio operato. Anche sotto questo aspetto comunque la nostra esperienza non può considerarsi un esempio, in quanto, essendo i dati funzionali ai tutorial, abbiamo condiviso sia i dati grezzi che quelli elaborati.

Concludiamo presentando un caso che si è verificato durante la realizzazione del progetto DADP e che è un chiaro esempio di sviluppo positivo della condivisione dei dati con ottica FS/OS. Per determinati tutorial avevamo bisogno di specifici dati, che la campagna di Aramus non era in grado di fornire. Si è sopperito a tale mancanza utilizzando materiale relativo ad un'altra campagna di scavo condotta dall'Università di Innsbruck in Iraq. Si è cioè creato un meccanismo virtuoso di contaminazione che richiama, ma in senso positivo, la definizione "virale" che Bill Gates attribuisce alla licenza "GPL".

\section{Conclusioni}

Vorremmo terminare fornendo brevemente alcune informazioni tecniche riguardo al progetto, che rimane (e rimarrà anche in futuro) un "work in progress", soggetto a migliorie e modifiche, necessarie a mantenere un prodotto aggiornato. 
L'attuale sito in cui si possono trovare i tutorial è ubicato al seguente indirizzo: http://vai.uibk.ac.at/dadp/.

I testi saranno disponibili in almeno tre lingue (inglese, tedesco e italiano), ma qualsiasi ulteriore traduzione è bene accetta. Ogni tutorial riporterà i nomi degli autori principali e di quelli di ogni contributo (modifiche, aggiornamenti, correzioni, ecc...), oltre naturalmente a quelli dei traduttori.

Attualmente sono previsti una ventina di tutorial (tabella 1) su argomenti tecnici riguardanti l'utilizzo di software libero, ma ogni testo attinente la disciplina archeologica è ovviamente il benvenuto. In futuro si spera che il sistema wiki diventi un contenitore non solo di testi, ma anche di altri tipi di risorse (filmati, software, dati, informazioni riguardanti scavi, ecc...).

Almeno inizialmente i contributi esterni verranno sottoposti ad una valutazione prima di essere integrati nel sistema, per evitare contenuti inappropriati; verrà adottato un modello di sviluppo simile a quello di tanti software liberi come GRASS, con una chiave d'accesso per le modifiche da rilasciare agli autori interessati a contribuire al progetto.

Chiunque voglia integrare il progetto lo può fare scrivendo ad uno dei seguenti indirizzi: luca.bezzi@arc-team.com, alessandro.bezzi@arc-team.com.

\section{Notes}

Arc-Team s.n.c.

† Dipartimento di Orientalistica e Storia Antica, Università di Innsbruck

${ }^{1}$ Sandra Heinsch e Walter Kuntner.

${ }^{2}$ Haik Avetisyan.

${ }^{3}$ Pavel Avetisyan.

${ }^{4}$ Lindsay e Smith 2006, p. 173.

${ }^{5}$ Khanzadyan 1979.

${ }^{6}$ P. Avetisyan e Bobokhyan 2008.

${ }^{7}$ H. Avetisyan 2001, pp. 37-50

${ }^{8}$ Smith e Kafadarian 1996, p. 36.

${ }^{9}$ H. Avetisyan e Allinger-Csollich 2006.

${ }^{10}$ Kuntner, Heinsch e H. Avetisyan in press.

${ }^{11}$ http://www.archeos.eu/

12 http://vai.uibk.ac.at/dadp/

13 Open Archaeology (http://www.arc-team.com)

14 “... aveva negli occhi la gelosia e il sospetto dell'archeologo..." Robert Byron, La via per l'Oxiana.

15 Si veda a tal proposito il tutorial riguardante la creazione di fotomosaici georeferenziati.

16 Supporti essenziali in un progetto didattico. 


\section{Riferimenti bibliografici}

Avetisyan, H. (2001). Aragats (Excavations of the urartian fortress). Yerevan.

Avetisyan, H. e W. Allinger-Csollich (2006). «The Fortress of Aramus: Preliminary Report of Excavations in 2004 and 2005». In: Aramazd. Armenian Journal of Near Eastern Studies 1, pp. 105-134.

Avetisyan, P. e A. Bobokhyan (2008). «The Pottery Traditions of Armenian Middle to Late Bronze Age 'Transition' in the Context of Bronze and Iron Age Periodization». In: Ceramics in Transitions: Chalcolithic through Iron Age in the Highlands of the Southern Caucasus and Anatolia, Conference held at New York in November 2004. A cura di Rubinson K. e Sagona A. Ancient Near Eastern Studies Supplement 27. Leuven, pp. 123-183.

Khanzadyan, E.V. (1979). Elar-Darani. Yerevan.

Kuntner, W., S. Heinsch e H. Avetisyan (in press). «The Fortress of Aramus in Achaemenid Times». In: Persepolis and his Settlements. Territorial System and Ideology in the Achaemenid State. Proceedings of the conference held in Viterbo, 16-17th December 2010. A cura di G. P. Basello e V. Rossi.

Lindsay, I. e A.T. Smith (2006). "A History of Archaeological Practices in Armenia and the South Caucasus». In: Journal of Field Archaeology 31.2, pp. 165-184.

Smith, A.T. e K. Kafadarian (1996). «New Plans of Early Iron Age and Urartian Fortresses in Armenia: A Preliminary Report on the Ancient Landscapes Project». In: Iran 34, pp. 23-37. 


\begin{tabular}{|c|c|c|}
\hline Argomento & Titolo & Programmi \\
\hline $\begin{array}{l}\text { Open Source e Free } \\
\text { Software }\end{array}$ & $\begin{array}{l}\text { Introduzione generale sui concetti } \\
\text { di Open Source e Free Software }\end{array}$ & GNU/Linux \\
\hline OS/FS in archeologia & $\begin{array}{l}\text { Introduzione ad ArcheOS } \\
\text { (applicazioni utili in archeologia) }\end{array}$ & ArcheOS \\
\hline GIS (2D) - scavo & $\begin{array}{l}\text { Elaborazione di coordinate e loro } \\
\text { importazione in un GIS }\end{array}$ & $\begin{array}{l}\text { Kwrite, Open- } \\
\text { Jump }\end{array}$ \\
\hline GIS (2D) - scavo & $\begin{array}{l}\text { Creazione di fotomosaici georefe- } \\
\text { renziati }\end{array}$ & $\begin{array}{l}\text { GRASS, efoto, } \\
\text { GIMP }\end{array}$ \\
\hline GIS (2D) - scavo & Vettorializzazione di fotomosaici & OpenJump \\
\hline GIS (2D) - scavo & $\begin{array}{l}\text { Gestione di un progetto in Open- } \\
\text { Jump con reperti e creazione di un } \\
\text { database }\end{array}$ & OpenJump \\
\hline CAD - scavo & $\begin{array}{l}\text { Creazione di un sistema di } \\
\text { coordinate }\end{array}$ & QCAD \\
\hline CAD - scavo & Digitalizzazione di un fotomosaico & QCAD \\
\hline GIS (3D) - scavo/studio & Creazione di un DEM & GRASS \\
\hline GIS (3D) - studio & $\begin{array}{l}\text { Analisi territoriali (slope, aspect, } \\
\text { contour, viewshed, ...) }\end{array}$ & GRASS \\
\hline GIS (3D) - studio & Cost analisys & GRASS, SAGA \\
\hline GIS (2D) & Visualizzazione dei dati & QGIS, GRASS \\
\hline Grafica (3D) & $\begin{array}{l}\text { Ricostruzione di vasi e solidi di } \\
\text { rotazione }\end{array}$ & Blender \\
\hline Database & Creazione di un database & $\begin{array}{l}\text { PostgreSQL, } \\
\text { PHPPgAdmin }\end{array}$ \\
\hline Database & $\begin{array}{l}\text { Creazione di una scheda US } \\
\text { (recording sheet) }\end{array}$ & $\begin{array}{l}\text { OpenOffice.org } \\
\text { Base }\end{array}$ \\
\hline $\begin{array}{l}\text { Stereorestituzione } \\
\text { fotogrammetrica }\end{array}$ & Ricostruzione di una struttura & Stereo \\
\hline Voxel & $\begin{array}{l}\text { Ricostruzione di un deposito } \\
\text { stratigrafico }\end{array}$ & GRASS, Paraview \\
\hline WebGIS & Creazione di un WebGIS & $\begin{array}{l}\text { MapServer, } \\
\text { MapLab, Pmapper }\end{array}$ \\
\hline Grafica 3D & Introduzione generale & Blender \\
\hline $\begin{array}{l}\text { Stereorestituzione } \\
\text { fotogrammetrica }\end{array}$ & $\begin{array}{l}\text { Ricostruzione di un DEM da una } \\
\text { coppia di foto stereo }\end{array}$ & efoto \\
\hline $\begin{array}{l}\text { Elaborazione di dati da } \\
\text { laserscan }\end{array}$ & Gestione di nuvole di punti & Scanalyze \\
\hline $\begin{array}{l}\text { Webpublishing e } \\
\text { condivisione di dati }\end{array}$ & $\begin{array}{l}\text { Creazione di un "finto 3d" e di una } \\
\text { pagina HTML }\end{array}$ & $3 \mathrm{DNP}$ \\
\hline Grafica vettoriale & $\begin{array}{l}\text { Introduzione generale (Harris } \\
\text { Matrix, ...) }\end{array}$ & Inkscape \\
\hline Grafica raster & Introduzione generale & Gimp \\
\hline
\end{tabular}

Tabella 1: Progetto tutorial di archeologia e Open Source - Free Software 


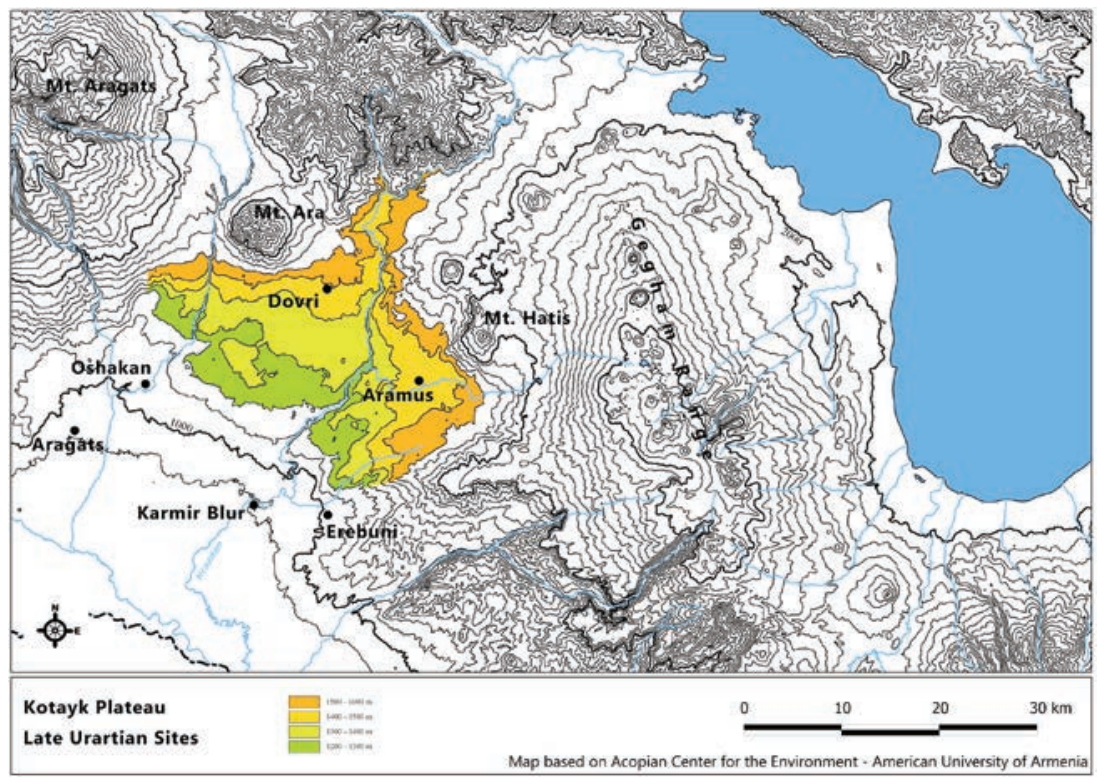

Figura 1: Localizzazione geografica di Aramus

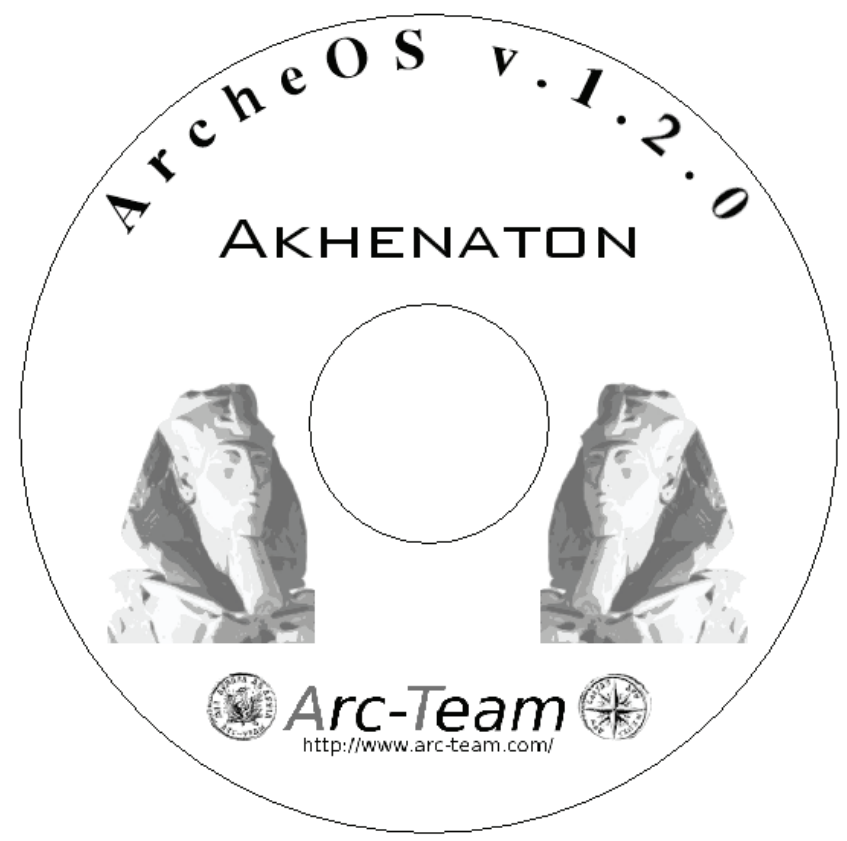

Figura 2: La distribuzione GNU/Linux ArcheOS 


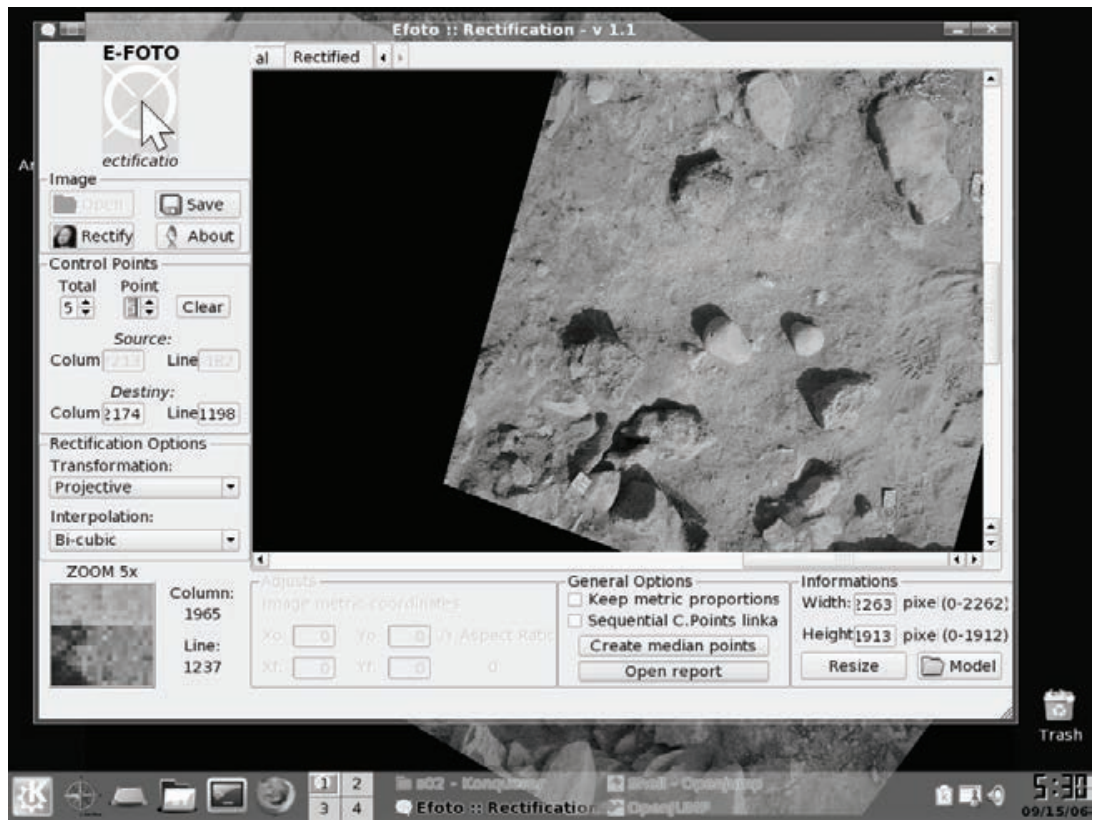

Figura 3: L'interfaccia di e-foto

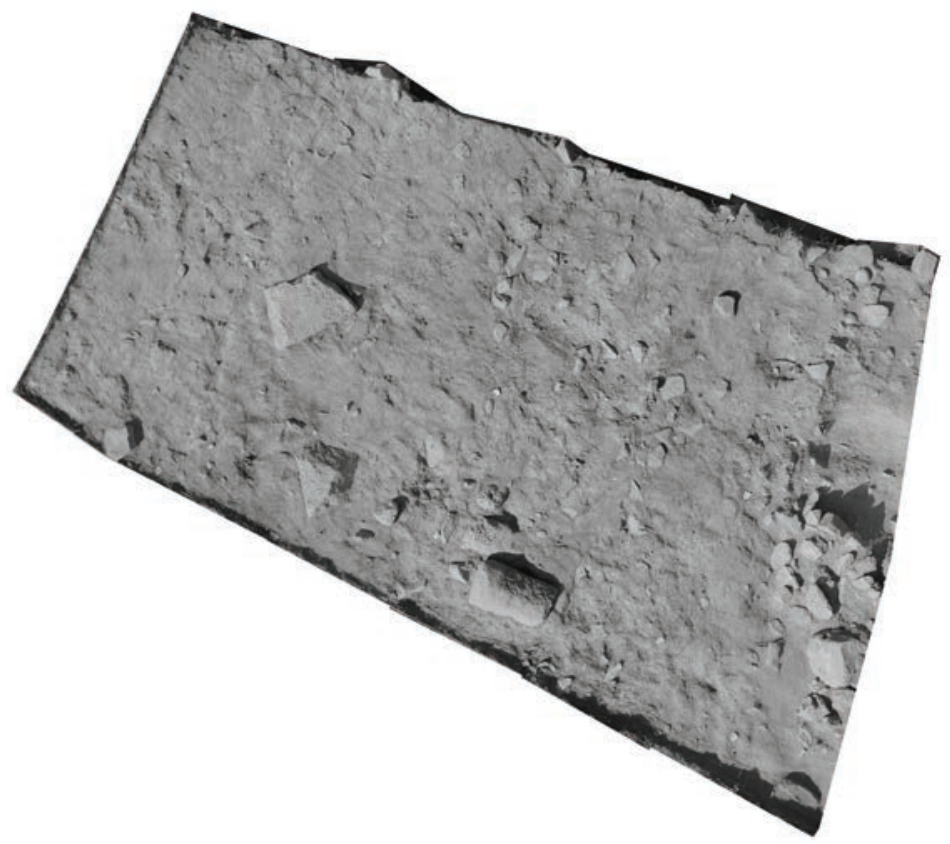

Figura 4: Metodo per il fotomosaico 